\title{
(6) OPEN ACCESS \\ Autism: the management and support of children and young people on the autism spectrum (NICE Clinical Guideline 170)
}

\section{Belinda H A Crowe, ${ }^{1}$ Alison T Salt ${ }^{1,2}$}

${ }^{1}$ The Wolfson Neurodisability Service, Great Ormond Street Hospital for Children NHS Foundation Trust, London, UK ${ }^{2}$ Institute of Child Health, University College London, London, UK

\section{Correspondence to} Dr Alison Salt, The Wolfson Neurodisability Service, Level 10, Old Nurses Home Building, Great Ormond Street Hospital for Children, Great Ormond Street, London WC1N 3JH, UK; Alison.Salt@gosh.nhs.uk

Received 6 January 2014 Revised 24 March 2014 Accepted 2 April 2014 Published Online First 8 May 2014
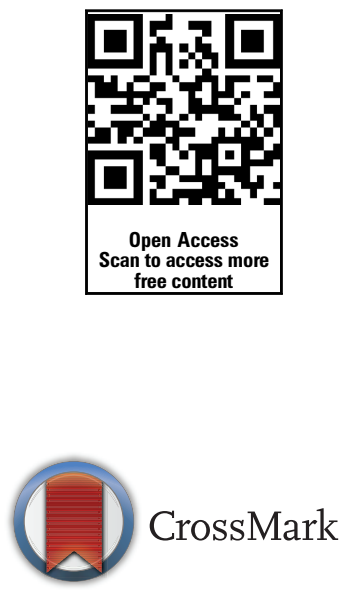

To cite: Crowe BHA, Salt AT. Arch Dis Child Educ Pract Ed 2015;100:20-23.

\section{INFORMATION ABOUT THE CURRENT GUIDELINE}

In August 2013 the National Institute for Health and Care Excellence (NICE) collaborated with the Social Care Institute for Excellence (SCIE) to publish a guideline entitled 'Autism: the management and support of children and young people on the autism spectrum'. It aims to summarise the different ways professionals can provide support, treatment and help for children and young people with autism across the full range of intellectual abilities, from birth until their 19th birthday. ${ }^{1}$ NICE commissioned the National Collaborating Centre for Mental Health to develop this guideline. A multidisciplinary Guideline Development Group comprising healthcare professionals, patients and carers reviewed the evidence and developed recommendations (box 1).

\section{PREVIOUS GUIDELINES}

This is the first national guideline to focus on the management and support of children and young people on the autism spectrum. It should be read in conjunction with 'Autism: recognition, referral and diagnosis of children and young people on the autism spectrum' (NICE clinical guideline 128) published in September 2011 and 'Autism: recognition, referral, diagnosis and management of adults on the autism spectrum' (NICE clinical guideline 142) published in June 2012. ${ }^{2}$

\section{KEY ISSUES ADDRESSED BY THE GUIDELINE}

- Access to health and social care services

- All children and young people with autism should have full access to services, including mental health services, regardless of intellectual ability or coexisting diagnosis.
- Knowledge and competence of health and social care professionals

- All professionals working with children and young people with autism should receive training in autism awareness and management, to include behaviour that challenges, risk assessment and communication.

- Adjusting the social and physical environment and processes of care

- Any negative environmental impacts should be assessed and minimised, for example, by providing meaningful visual supports, adapting personal space and considering sensory sensitivities to lighting, noise and the colour of surroundings.

- Processes of care should be adapted to meet individual needs, for example, by arranging appointments at the beginning/ end of the day to minimise waiting times and by providing single rooms for children requiring anaesthetics.

- Families and carers

- Families and carers should be offered an assessment of their own needs, which considers personal, social and emotional support, practical support to include short breaks, emergency plans and a plan for future care for the child or young person, including transition to adult services.

- Psychosocial interventions

- A specific social-communication intervention for the core features of autism should be considered, which includes play-based strategies with parents, carers and teachers to increase joint attention, engagement and reciprocal communication.

- Strategies should be adjusted to the individual's developmental level and aim to increase understanding of their patterns of communication and interaction. 
Box 1 Resources

NICE guidance and full guideline:

http://guidance.nice.org.uk/CG170

Information for the public:

http://publications.nice.org.uk/

managing-autism-in-children-and-young-people-ifp170

NICE Autism Pathway:

http://pathways.nice.org.uk/pathways/autism

The National Autistic Society-the leading UK charity for people with autism and their families:

http://www.autism.org.uk/

Contact A Family-a charity providing peer support for families of disabled children:

http://www.cafamily.org.uk/

- Interventions should be delivered by trained professionals and appropriately mediated by parents, carers, teachers or peers.

- Behaviour that challenges

- Factors that may increase the risk of behaviour that challenges should be assessed and addressed, for example, impairments in communication, coexisting physical/mental health disorders, the environment, changes in routine, onset of puberty, inadvertent reinforcement of behaviour that challenges or the absence of structure.

- If no trigger is identified, a psychosocial intervention should be offered as first-line treatment.

- Consider antipsychotic medication when psychosocial or other interventions are insufficient, or could not be delivered because of the severity of the behaviour (see box 2).

- Life skills

- Children and young people with autism should be offered support in developing coping strategies and skills to access community services, including public transport, employment and leisure facilities.

Box 2 Antipsychotic medication for behaviour that challenges

Medication should always be:

prescribed by a paediatrician or psychiatrist

- started at a low dose and using the minimum effective dose needed

- used with appropriate informed consent as currently prescribed off licence

- monitored with respect to effectiveness and side effects after 3-4 weeks and then, using an identified target behaviour,

- stopped if there is no indication of a clinically important response at 6 weeks.
Coexisting problems

- Psychosocial and pharmacological interventions for coexisting mental health or medical problems should be offered in line with previous NICE guidance, for example, attention deficit hyperactivity disorder (ADHD), conduct disorders, constipation, depression, epilepsy, obsessive-compulsive disorder, body dysmorphic disorder and post-traumatic stress disorder.

- Consider group or individual cognitive behavioural therapy (CBT) for children and young people with autism and anxiety who have the verbal and cognitive ability to engage.

- Consider adapting the method of CBT delivery, for example, greater use of written and visual information, emotion recognition training, a more cognitively concrete/structured approach and incorporation of the child or young person's special interests.

- Sleep problems

- Provide detailed assessment of sleep problems and appropriate management advice to children and young people with autism, their families and carers (see box 3).

- Transition to adult services

- Reassess young people with autism receiving input from child/mental health services at around age 14, to verify the need for continuing treatment into adulthood.

\section{Box 3 Sleep problems}

Identify and assess the nature of the problem, including:

$\checkmark$ bedtime routine

- sleep environment

- comorbidities, for example, hyperactivity, physical illness or discomfort

- individual factors, for example, problems at school

- the impact of sleep and behavioural problems on the family

- loud snoring, choking or pauses in breathing: refer to a specialist to assess for obstructive sleep apnoea if present.

Develop a sleep plan to address problems and establish a regular night-time pattern.

Ask parents to record a 2-week sleep diary and use this to modify the sleep plan, before considering medication.

If ongoing sleep problems have a negative impact on the child or family, offer medication alongside the sleep plan in consultation with a paediatrician/psychiatrist with expertise in autism/sleep medicine. Review regularly to ensure benefits outweigh side effects and risks.

If sleep problems persist, consider referral to a paediatric sleep specialist and overnight short breaks/other respite care repeated regularly. 
- Carry out a comprehensive assessment, including coexisting conditions, for example, depression, anxiety, ADHD and intellectual disability. ${ }^{3}$

- For those aged 16 or older with complex/severe needs, use the care programme approach (CPA) in England or care and treatment plans in Wales to aid transfer between services.

- Involve the young person in planning (and parents/ carers where appropriate) and provide information about adult services, including their right to a social care assessment at age 18 .

- Consider a formal multidisciplinary meeting with professionals from child and adult services to ensure continuity of care.

\section{WHAT DO I NEED TO KNOW?}

$\checkmark$ What should I not do?

- Do not use antipsychotics, antidepressants, anticonvulsants or exclusion diets (eg, gluten or casein-free diets) to manage the core features of autism.

- Do not use neurofeedback or auditory integration training to manage speech and language problems in autism.

- Do not routinely use a pharmacological intervention to aid sleep (see box 2).

- Do not use omega-3 fatty acids to manage sleep problems.

- Do not use secretin, chelation or hyperbaric oxygen therapy to manage autism in any context.

- What should I ensure I've started doing?

- Ensure full access to health and social care services, including mental health services, for all children and young people with autism.

- Provide the interventions recommended in this guideline to individuals with particular needs, for example,

\section{Box 4 What do I need to inform the families in my} care?

Provide ongoing tailored information, with details about:

- local and national organisations that provide peer support

autism courses

leisure activities

social support including access to benefits

- educational support

local services and treatments

transition to adult services.

Offer flexible support during times of increased need, for example, transitions such as the birth of a sibling, puberty or changing schools.

Regularly encourage and provide opportunities for children, young people and families to collaborate and be involved in shared decision making about their care, taking their views into account. looked-after children, those from immigrant groups or those with co-existing conditions such as mental health problems, through local autism teams.

- Assess co-existing mental health or medical problems and offer appropriate interventions in line with previous NICE guidance.

- Discuss help available locally with parents, siblings and carers (see boxes 1, 4 and 5).

- Start thinking about transition to adult services early.

What can I continue to do as before?

- Assess, manage and coordinate care for children and young people with autism through local specialist community-based multidisciplinary autism teams. ${ }^{2} 3$

- Allocate a case manager or key worker to manage and coordinate care, including transition, for every child or young person affected. ${ }^{2}$

- Refer children and young people to a regional or national autism service if there is a lack of local skills required to provide intervention and care for those with complex coexisting conditions, for example, severe sensory or motor impairment or mental health problems. $^{12}$

\section{UNRESOLVED CONTROVERSIES/RESEARCH RECOMMENDATIONS}

Areas of autism management that require further research to assess their clinical and cost-effectiveness include:

- A key worker approach

- Group-based parent training interventions to manage behaviour that challenges

- Sleep hygiene interventions or melatonin for sleep problems

- Pharmacological and psychosocial interventions for comorbid anxiety

\section{Box 5 Key points for the general paediatrician}

- Provide holistic care for children and young people with autism which is flexible and tailored to their individual needs.

> Treat coexisting medical or mental health problems appropriately, with input from mental health services.

- Offer psychosocial rather than pharmacological interventions first line for features of autism that impact negatively on quality of life, for example, social communication, sleep difficulties and behaviour that challenges.

- Work closely with colleagues in community paediatrics, neurodisability and sleep medicine to optimise patient care.

$>$ Remember that autism is a life-long condition and plan transition carefully.

- Ensure children, young people and families can access appropriate support. 
- Teacher, parent and peer-mediated psychosocial interventions to manage the core symptoms of autism in preschool children.

\section{Clinical bottom line}

Although there is currently no cure for autism, much can be done to optimise quality of life for the children, young people and families affected.

- This guideline takes a holistic approach to management using high quality evidence to ensure all those diagnosed with autism receive the best possible care.

Contributors BHAC prepared the first draft of the review and ATS advised on and jointly wrote subsequent revisions.

Competing interests None.

Provenance and peer review Commissioned; externally peer reviewed.
Open Access This is an Open Access article distributed in accordance with the Creative Commons Attribution Non Commercial (CC BY-NC 3.0) license, which permits others to distribute, remix, adapt, build upon this work noncommercially, and license their derivative works on different terms, provided the original work is properly cited and the use is non-commercial. See: http://creativecommons.org/licenses/by$\mathrm{nc} / 3.0 /$

\section{REFERENCES}

1 National Institute for Health and Care Excellence. Autism: the management and support of children and young people on the autism spectrum. CG 170. London: National Institute for Health and Care Excellence, 2013.

2 National Institute for Health and Care Excellence. Autism: recognition, referral and diagnosis of children and young people on the autism spectrum. CG128. London: National Institute for Health and Care Excellence, 2011.

3 National Institute for Health and Care Excellence. Autism: recognition, referral, diagnosis and management of adults on the autism spectrum. CG142. London: National Institute for Health and Care Excellence, 2012. 